\title{
Custom Total Knee Arthroplasty
}

\author{
Elliot Sappey-Marinier, Carsten Tibesku, \\ Tarik Ait Si Selmi, and Michel Bonnin
}

\section{Key Points}

1. Restore the native shape with an optimal bone implant fit.

2. Reproduce the native pre-arthritic joint line with a personalized alignment.

3 . New economic system using custom singleuse instruments.

As we reach the 50th anniversary of 'modern TKA', new technologies and new industrial processes render the manufacture of fully customized implants feasible. While this can be considered as a technological breakthrough, addressing several limitations of TKA, we may question whether this costly technology is worthwhile and beneficial for patients. Considering the wide range of TKA sizes now available-sometimes having millimetric-size increments—do we really need customized implants to reproduce the native anatomy?

E. Sappey-Marinier · T. A. S. Selmi · M. Bonnin $(\bowtie)$ Centre Orthopédique Santy, Jean Mermoz Private Hospital, Lyon, France

C. Tibesku

KniePraxis, Straubing, Germany

e-mail: carsten@tibesku.de

\subsection{Why Custom Total Knee Arthroplasty?}

\subsubsection{A Brief History of TKA}

During the first half of the twentieth century, the pioneers of arthroplasty surgery tested surgical procedures for arthritic knees, which could be considered as 'resurfacing procedures' using soft tissue or chromium-cobalt interposition [1]. Inspired by the success of Smith-Petersen [2] with mould arthroplasty of the hip, Campbell and Boyd performed the first arthroplasty of the knee [3]. The advent of 'modern TKA' in the early 1970s introduced standardization, precision and reproducibility of surgical techniques and manufacturing processes, but abandoned the concept of personalized resurfacing. Due to the limited number of sizes available (only one femoral size existed during the first decade of total condylar knee arthroplasty) [4], optimizing a bone implant fit was challenging. During the 1980s and 1990s, the range of sizes increased, but only proportional to the original designs, assuming that all human knees had the same shape. It was only in the early 2000s that morphologic variability was investigated through the aspect ratio [5] and manufacturers developed narrow versions in their range of femurs, known as 'gender knees'. 


\subsubsection{The Limits of Contemporary TKA}

Nowadays, surgeons can choose components from a wide range of sizes, including standard and narrow and sometimes asymmetric tibia. However, anatomic variations are not limited to large or narrow, but also include several other features, such as the trapezoidicity of the distal femur [6], the condylar radii of curvature [7], joint-line obliquity [8] and the shape of the trochlea and tibial plateaus [9]. The observed variability in morphotypes echoes the words of John Insall who warned that "care must be taken in describing what is "normal" because of significant individual variations' and those of Werner Müller who pointed out that 'nothing is as constant as the variability of anatomy'. Therefore, the size and shape ranges used in standard TKA hardly cover the variability of the human knee, and oversizing has been reported in up to $76 \%$ on the femur and up to $90 \%$ on the tibia after TKA. It has been also demonstrated that any overhang of the implants increases the risk of residual pain and stiffness and jeopardizes functional outcomes [10-12].

Moreover, because the soft tissue envelope is non-extensible, implantation of mechanically aligned prostheses causes ligament imbalance, patellar maltracking and stiffness. These are tackled by the use of technical tricks such as ligament releases [13], external rotation of the femoral component [14] and kinematic alignment [15], all of which are 'palliative solutions' compensating for the non-anatomic shape of the implants and modification of the native alignment. It is therefore important to understand that TKA alignment and implant design are inter-related and cannot be considered separately.

\subsubsection{Alignment Strategy in TKA}

In the early days of TKA, the so-called mechanical alignment (MA) was favoured, aiming for a straight leg axis of $180^{\circ}$ (neutral alignment), obtained via orthogonal cuts. A perfectly straight $180^{\circ}$ leg does not mirror the average alignment, but was chosen for reasons of reproducibility and load distribution, to minimize polyethylene wear and implant loosening [16]. The mean native joint line obliquity (JLO) is $3^{\circ}$, with large inter-individual variations, comprising the mechanical lateral distal femoral angle (mLDFA), the mechanical medial proximal tibial angle (mMPTA) and the joint line convergence. The native JLO is rarely reproduced with classic orthogonal cuts, which results in asymmetric bone resections and therefore 'iatrogenic laxity'. The anatomic alignment (AA) technique for $T K A$ still aims at a neutral $\left(180^{\circ}\right)$ alignment but via slightly oblique cuts $\left(3^{\circ}\right)$, which reproduces the average JLO value. The kinematic alignment (KA) technique for TKA, introduced later, aims to adapt the position of the implant to the soft tissue envelope, thereby restoring the native tri-dimensional alignment of the lower limb. Whatever the chosen alignment technique, a one geometrical implant design may cause bone implant mismatches when used in the wide range of human knee anatomies. So personalized implant orientation with KA may benefit to be linked to implant customization.

\subsubsection{Are Patients Fully Satisfied with Standard TKA?}

Despite the increasing survival of TKAs, due to innovations in biomaterials, design and surgical techniques, the satisfaction rate following TKA reported in the literature varies from $75 \%$ to $89 \%$, with three main influencing factors: residual pain, functional outcome and preoperative expectations [17-20]. In a multicentre series of 347 non-selected TKA patients using various implants [17], we observed that only $62 \%$ of our patients were totally pain free during gait and $35 \%$ while climbing or descending stairs and $40 \%$ complained of pain while running. Only $48 \%$ of the patients declared being 'very satisfied' with the procedure, and 68\% considered their operated knee to be 'normal for their age'. 


\subsection{The Need for Custom TKA}

The Origin ${ }^{\circledR}$ custom TKA (Symbios, Yverdonles-Bains, Switzerland) was developed between 2012 and 2017 and is CE marked since 2018. This system was conceived and designed to reproduce the native (pre-arthritic) anatomy of the knee, using a single-use custom instrumentation. The main aims are:

1. To optimize bone implant fit and avoid prosthetic overhang or under-coverage.

2. To improve ligament balancing by avoiding resection laxity due to asymmetric bone cuts.

3. To improve mid-flexion stability and kinematics by restoring the native radii of curvature.

4. To improve patellofemoral tracking by restoring the native femoral torsion and customized trochlea.

5. To facilitate restoration of the native prearthritic limb alignment.

Its production is based on a classic process, the chromium-cobalt femoral implant being made by standard casting, followed by machining and polishing. The tibial baseplate is made of titanium.

\subsection{Design Rationale of the Origin Implant}

The Origin ${ }^{\circledR}$ prosthesis is postero-stabilized, with a proportional post-cam system that engages beyond $60^{\circ}$ of knee flexion. The intercondylar box is proportional, in order to minimize bone sacrifice. Between $0^{\circ}$ and $60^{\circ}$ of knee flexion, anteroposterior stability relies on the shape of the polyethylene with a specific anterior ultracongruency. Most of the prosthetic designs retaining or sacrificing the PCL do not stabilize the femur adequately during flexion and allow a paradoxical femoral anterior sliding, which constrains the patella and reduces the quadriceps lever arm.

The femoral component reproduces the shape of the native femur, in terms of contours, radii of curvature and joint line obliquity. Because the implant and instrumentation are devised to reproduce the natural shape of the distal femur, no additional rotation is required during implantation, and the design is linked to the alignment. Therefore, no intraoperative modification of the femoral cuts or femoral rotation need be considered. The prosthetic trochlea is designed to match the shape of the native patella and to maintain its native alignment, with soft edges to avoid patellofemoral crepitus. In cases of trochlear or patellar dysplasia, the femoral trochlea implant is designed as a standard trochlea.

The tibial baseplate is asymmetric and reproduces the contours of the native plateaus, facilitating rotational positioning of the implant after bone resection. The rotation of the tibia matches the transverse tibial axis, defined by the line joining the centres of each plateau. The tibial slope is maintained in a range of $2^{\circ}-5^{\circ}$ to avoid anteroposterior instability. The tibial keel is aligned medio-laterally with the axis of the tibial metaphysis, which corresponds non-systematically to the baseplate centre. The coronal alignment of the tibial cut is maintained in $\pm 3^{\circ}$ range from $90^{\circ}$.

\subsection{What Is the Process?}

The design and manufacturing process of the Origin ${ }^{\circledR}$ custom TKA takes 6 weeks and requires cooperation between the surgeon and engineers. The design is based on a three-dimensional analysis of the bony anatomy of the arthritic deformities and limb alignment, based on a preoperative CT scan, using a special radiographic protocol, including the knee, hip and ankle joint. The DICOM files are collected and sent electronically to the engineering team through a secured 'Symbios box'. 3D analysis is performed with Knee-Plan ${ }^{\circledR}$ software (Symbios, Yverdon-lesBains, Switzerland) (Fig. 22.1).

Additional clinical (range of motion of the knee, reducibility of the deformity) and radiographic information (dynamic varus-valgus XR and long leg standing XR) may also be useful. The engineering process requires several steps: 

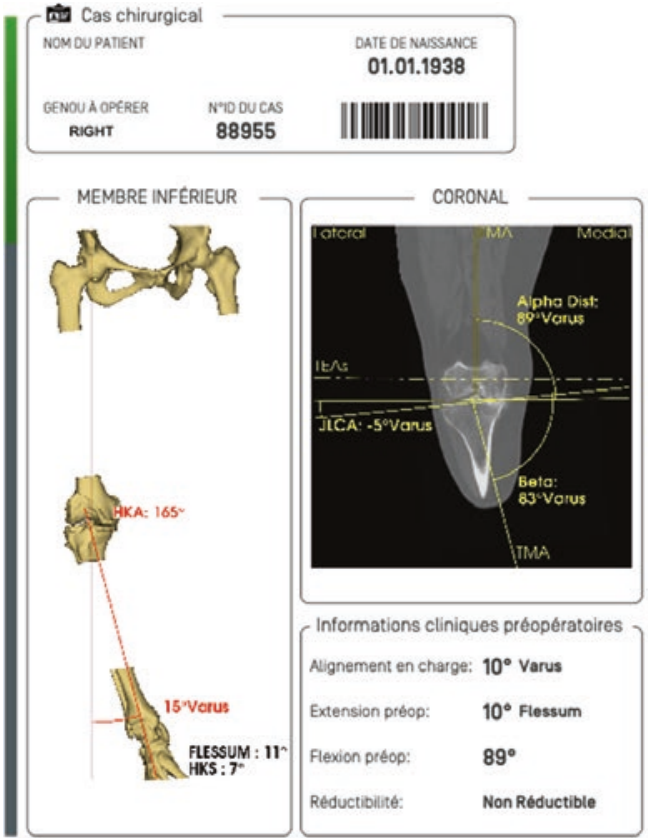

version 1

10.10.2018 16:20:44

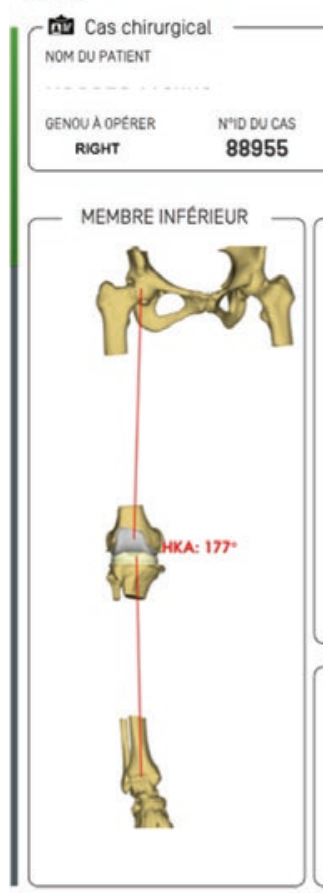

version 1 page $2 / 4$

Commentaires planificateur

\section{KNEE-PLAN ${ }^{\circledR}$ \\ PREOPERATIVE ANALYSIS}

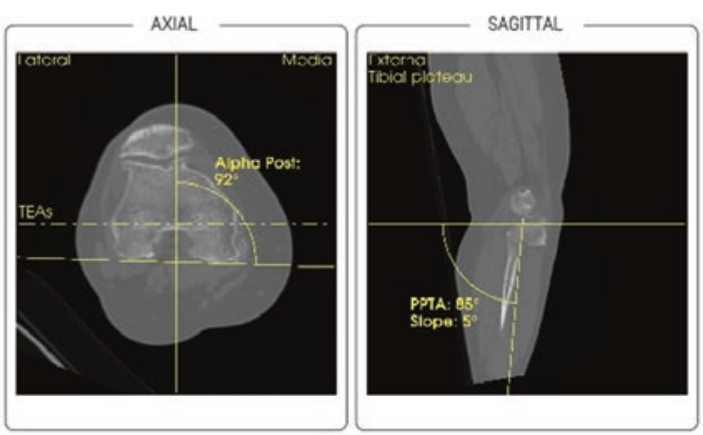

Commentaires du chirurgien symbios

\section{KNEE-PLAN ${ }^{\circledR}$ \\ PLANNING SUMMARY}

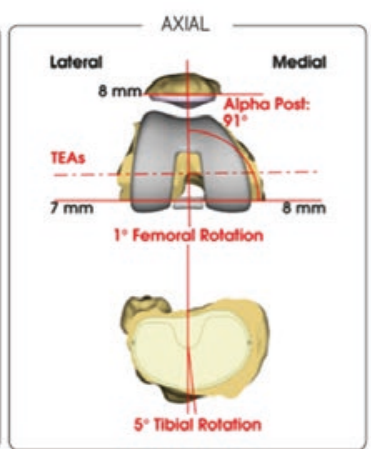

- SAgItTAL

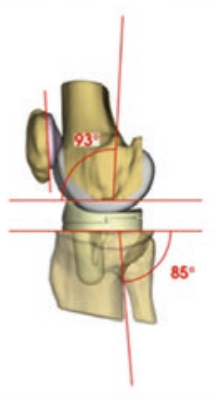

Fig. 22.1 Knee-plan pre-operative analysis 
1. Semi-automated 3D reconstruction and segmentation of the distal femur, proximal tibia and patella.

2. Planning is done with Knee-Plan ${ }^{\circledR}$ software. From raw images, the alignment and bone wear are analysed, and the native (prearthritic) alignment is deduced. The realignment strategy is then established, including the level and orientations of the bone cuts. The aim is to reproduce the native alignment called the Origin Alignment ${ }^{\odot}$, in a range of $\pm 3^{\circ}$ from $180^{\circ}$, with a reproduction of the joint line obliquity in a range of $\pm 5^{\circ}$, in accordance with the restricted kinematic alignment protocol from Vendittoli (Chap. 17).

3. The design of the definitive implants, trial implants and custom instruments is then finalized, using SolidWorks ${ }^{\circledR}$ software (Dassault Systèmes, Vélizy-Villacoublay, France).

4. Operative planning and the implant design are then validated online by the surgeon.

5. Manufacturing of the definitive implants is then finalized, using 'pre-shapes', previously manufactured with classic chromium-cobalt casting technology for the femur and titanium (Ta6V) for the tibial baseplate. From a wide range of 'pre-shapes', the next largest sized pre-form is chosen, and final customization is done using automated quick milling technology to reproduce the shape of the native pre-arthritic bone.

6. The custom guides are made with additive manufacturing technology using polyamide (PA2200).

7. Implants and instruments are then assembled into a single box and sent directly to the hospital.

\subsection{Which Alignment Strategy?}

1. The Origin ${ }^{\circledR}$ alignment aims to reproduce both native (pre-arthritic) alignment and joint line obliquity, based on the preoperative CT scan with 3D reconstruction of the hip, knee and ankle. Bone wear and arthritic deformity are assessed and corrected during 3D reconstruc- tion. The mLDFA is recreated by reconstruction of the native femoral surface. The mMPTA is measured and recreated by a combination of bone cut adjustment (up to $3^{\circ}$ ) and an asymmetric polyethylene inlay (up to $2^{\circ}$ ), similar to the restricted kinematic alignment protocol from Vendittoli (Chap. 17).

The native alignment, also known as constitutional alignment, is determined from a combination of (1) the knee morphology obtained from a CT scan; (2) clinical data, notably the reducibility of the axis deviation and (3) the weight-bearing axis from a whole-leg standing radiograph. This Origin Alignment ${ }^{\oplus}$ does not seek to change the axis to $180^{\circ}$, but to restore the native alignment.

At this stage, there are certain limitations in the reconstruction of both native alignment and joint line obliquity. While it has been demonstrated that restoration of the native JLO in patients with constitutional varus decreases peak knee adduction [21], the Origin Alignment ${ }^{\odot}$ remains within safe limits in terms of tribology, fixation with a JLO range of $\pm 5^{\circ}$ and a postoperative alignment-range of $\pm 3^{\circ}$. Allowing for these limitations, approximately $75 \%$ of knee OA patients are suitable for Origin Alignment ${ }^{\odot}$ [22]. The other cases are managed individually according to the surgeon's preference.

\subsection{Surgical Technique}

All instruments are single-use custom tools and fit in a single box weighing $3 \mathrm{~kg}$.

\subsubsection{Femoral Preparation}

In this technique, the femoral preparation is done first (Fig. 22.2), because the femur is the driver of the knee kinematics. The first surgical step is to remove the remnants of cartilage, using electrocautery, a curette or a scalpel blade, in the areas of contact of the cutting block. The first femoral jig is secured to the bone with pins as soon 

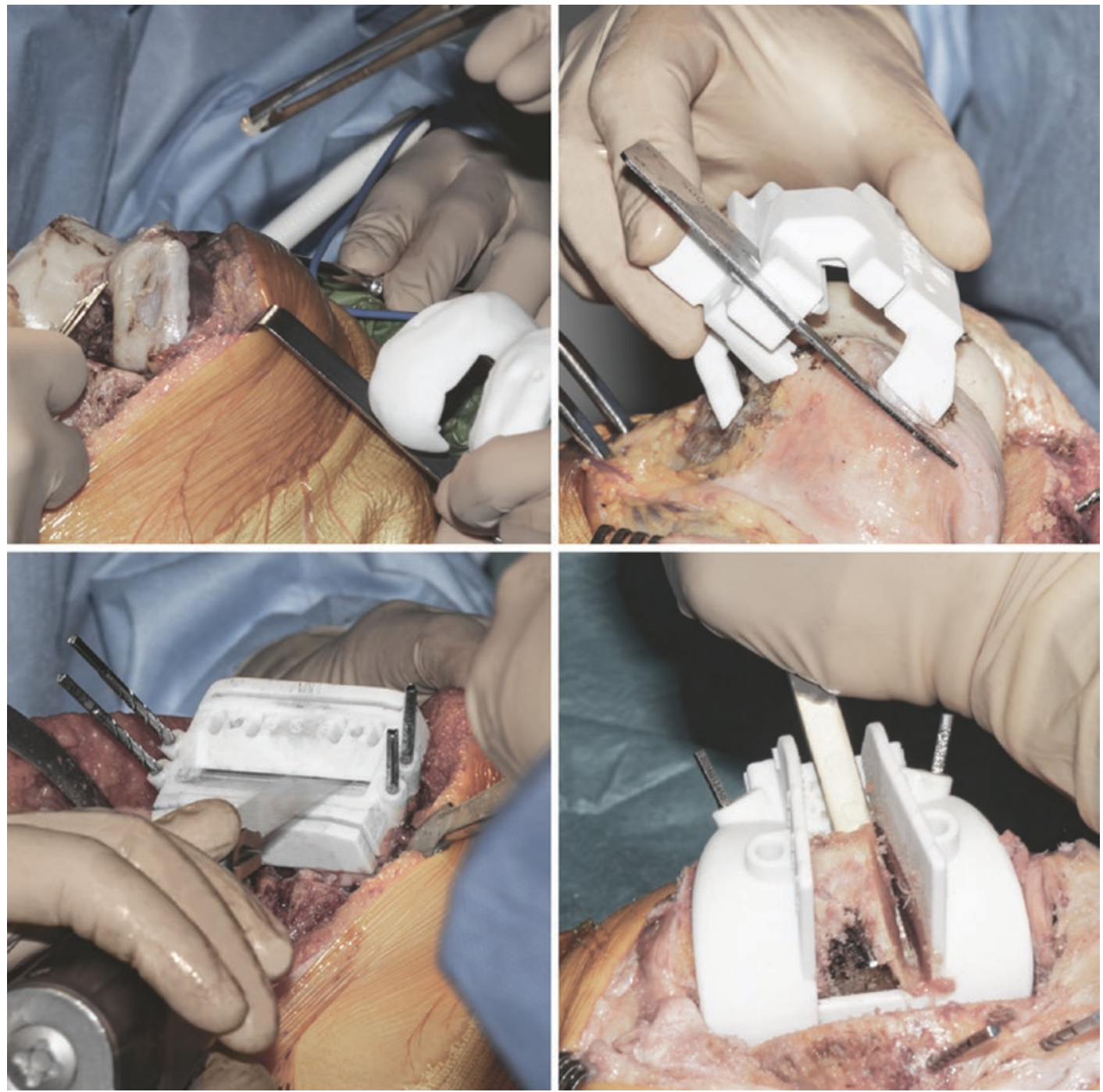

Fig. 22.2 Different steps of the femoral preparation

as a unique and stable position has been found for it, and cuts are performed with an oscillating saw. No femoral recuts are needed as the aim of the procedure is to reproduce precisely the shape of the distal condyles. The 'four in one' femoral cutting guide (second femoral jig) is positioned on the distal resected femur without any adjustment in size or rotation. The concept of femoral rotation has here no meaning because the femoral implant reproduces the shape of the distal femur and the thickness of the polyethylene reproduces the native joint line obliquity. Resection of the intercondylar femoral notch is guided by the third femoral jig. The medio-lateral contour of the bloc-specific to the patient-matches the bony contours of the femur.

The trial femoral component is positioned on the distal femur, and flexion/extension motion and valgus/varus stressing are done to assess the amount of bone wear and the level of the tibia cut.

\subsubsection{Tibial Preparation}

After removal of the cartilage remnants and osteophytes, the tibial jig is positioned on the 

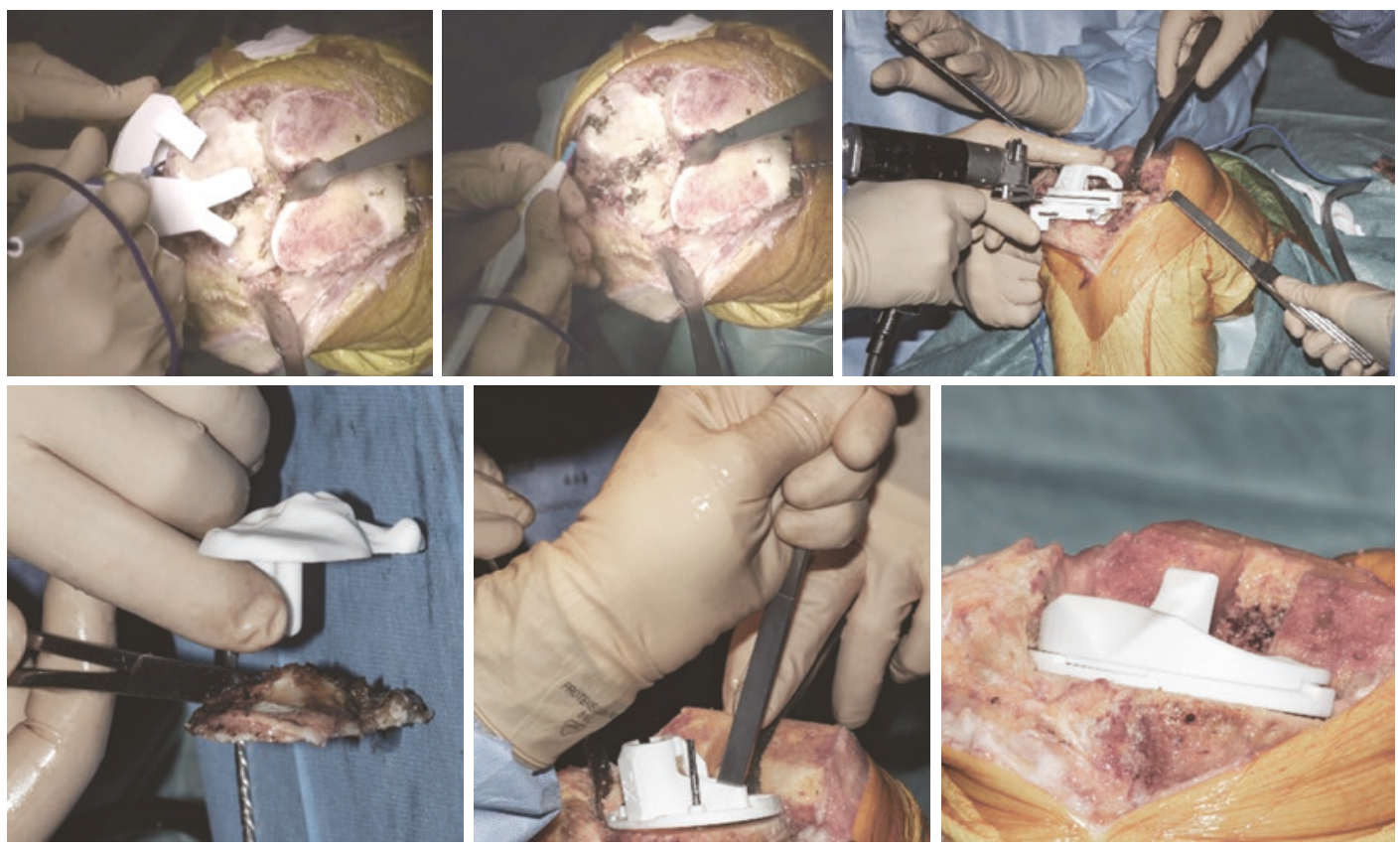

Fig. 22.3 Different steps of the tibial preparation

plateaus and secured with pins once the position is stable (Fig. 22.3). To meet the planned cut's orientation, the extramedullary alignment control rod must be centred medio-laterally to the centre of the ankle joint.

The resection provided by the tibial guide is a pre-cut of $-2 \mathrm{~mm}$ (minus $2 \mathrm{~mm}$ ) with respect to the planning. In most cases, a $+2 \mathrm{~mm}$ recut must be done as a second step (corresponding to the planned resection), after checking the stability with the 'Bone Balancer' (trial femoral implants with a floating tibial component). This recut is guided by the 're-cutting guide'. In some knees with laxity, if the ligament balancing is correct after the first tibial cut (pre-cut), the additional $+2 \mathrm{~mm}$ recut can be skipped. Conversely, in some stiff knees, an additional re-cut ( $+4 \mathrm{~mm}$ from the first cut) may be necessary, using the same 'recutting guide'.

After obtaining a good range of motion, with a balanced knee, controlled with the 'Bone Balancer' (medio-lateral stability with a slight residual varus-valgus laxity), the definitive tibial preparation is performed. The custom tibial baseplate (keel position and contouring are patient specific) is then fixed on the resected tibia surface, and the central peg and fins are prepared.

\subsubsection{Final Implantation}

The trochlea of the Origin ${ }^{\circledR}$ prosthesis is designed to match the shape of the native patella (anatomic trochlea), so patellar resurfacing is not required, but is recommended in cases of severe patellar osteoarthritis.

Once all bone surfaces have been prepared, the implants are cemented, firstly with the tibia and lastly with the femoral component (Fig. 22.4). Standard closure and dressing are then performed.

\subsubsection{Postoperative Care}

Physiotherapy begins a few hours after surgery, with immediate full weight bearing, using crutches only for safety and without any flexion or extension restrictions. Rehabilitation is based mostly on self-rehabilitation performed under the supervision of a physiotherapist, 

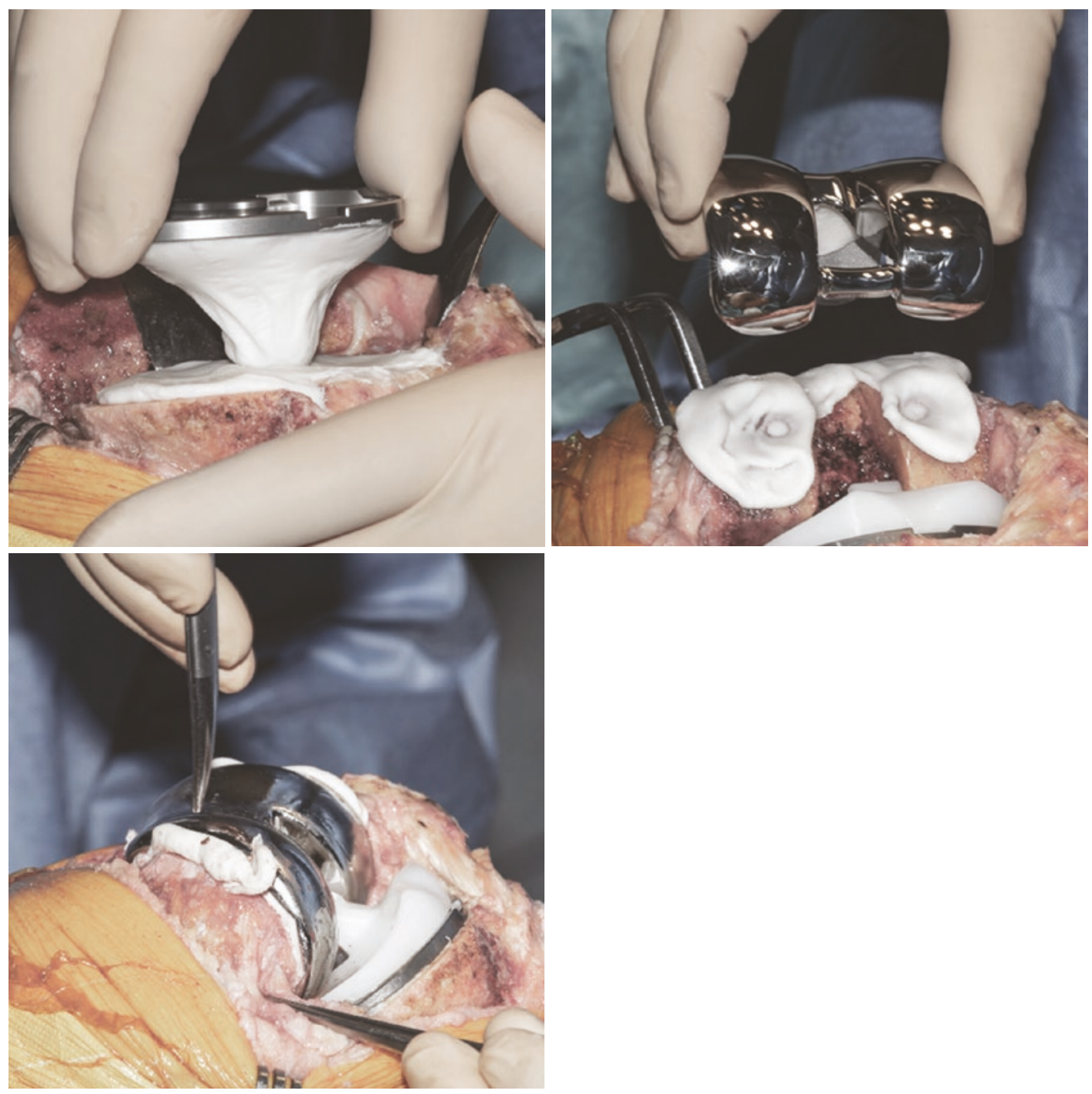

Fig. 22.4 Final cemented implantation

avoiding any active muscle strengthening earlier than 4 months postoperative.

\subsection{What Are the Potential Benefits of Custom TKA?}

\subsubsection{Patient Benefits}

This technology is based on the theory that many unsatisfactory outcomes and/or residual pain after TKA can be attributed to a lack of anatomic restoration, hardly identifiable by medical examination. Residual pain, stiffness and laxity are often secondary effects of incorrect sizing $[11,12]$ or malrotation [14]. Furthermore, asymmetric resections due to alignment strategy and the non-anatomic shape of the implants cause 'iatrogenic' laxities or stiffness. We therefore believe that an optimal restoration of the native anatomy-including limb alignment-may help improve functional outcomes in TKA. Also, customization of the bone cuts and implants allows engineers to minimize, as much as possible, the thickness and weight of the implants and the number of bone resections needed. 
It is worth noting that custom cutting guides or navigation systems, generally used in standard implants, failed to demonstrate clear benefits in terms of outcomes and patient satisfaction. Similarly, robotic surgery might be more reliable and precise in the future, but will not solve the major difficulties due to the non-anatomic design of the implant itself. We strongly believe that TKA needs improvements to its three main pillars: (1) definition of a personalized alignment strategy; (2) improvement of surgical precision with new technology, such as robotics and (3) restoring the native knee anatomy using custom implants.

\subsubsection{Benefits for the Surgeon}

Customized TKA offers many advantages to the surgeon. Firstly, the surgical process is easier, because conservation or restoration of the native anatomy automatically addresses many surgical difficulties: (1) femoral and tibial rotations are adjusted during the design stage, and adjustments to component positioning are required; (2) balancing is easier, particularly at mid-flexion, due to the conservation of the condylar curvature radii and the JLO; (3) no size adjustments are needed as the bone implant fit is optimized. Secondly, planning is defined preoperatively in terms of alignment and implant positioning, which safeguards the surgeon. Thirdly, this technology may help in certain difficult cases such as (1) patients with post-traumatic extra-articular deformities where correction of the deformity is easier; (2) patients with inextractible hardware close to the joint surface, where the instruments and the implant are designed to avoid impingement; (3) patients with multioperated or previously infected bones, because no bone catheterism is required; and (4) patients with extreme anatomy, where implantation of standard TKA may be challenging.

\subsubsection{Hospital Benefits}

This technology is valuable for the hospital management, simplifying the process in the theatre, with a single box including the implants and instruments, all tailored for the patient. It eliminates the need for large inventories of implants and instrumentation trays. Finally, this technology dramatically decreases the need for sterilization, with a major economic impact (cost) and also ecological consequences (water used for sterilization).

\section{References}

1. Campbell W. Interposition of vitallium plates in arthroplasties of the knee. Am J Surg. 1940;47(3):639-41.

2. Smith-Petersen MN. Evolution of mould arthroplasty of the hip joint. J Bone Joint Surg Br. 1948;30B(1):59-75.

3. Jones WN. Mold arthroplasty of the knee joint. Clin Orthop Relat Res. 1969;66:82-9.

4. Insall JN, Hood RW, Flawn LB, Sullivan DJ. The total condylar knee prosthesis in gonarthrosis. A five to nine-year follow-up of the first one hundred consecutive replacements. J Bone Joint Surg Am. 1983;65(5):619-28.

5. Hitt K, Shurman JR 2nd, Greene K, McCarthy J, Moskal J, Hoeman T, Mont MA. Anthropometric measurements of the human knee: correlation to the sizing of current knee arthroplasty systems. J Bone Joint Surg Am. 2003;85-A(Suppl 4):115-22.

6. Bonnin MP, Saffarini M, Bossard N, Dantony E, Victor J. Morphometric analysis of the distal femur in total knee arthroplasty and native knees. Bone Joint J. 2016;98-B(1):49-57.

7. Howell SM, Howell SJ, Hull ML. Assessment of the radii of the medial and lateral femoral condyles in varus and valgus knees with osteoarthritis. J Bone Joint Surg Am. 2010;92(1):98-104.

8. Bellemans J, Colyn W, Vandenneucker H, Victor J. The Chitranjan Ranawat award: is neutral mechanical alignment normal for all patients? The concept of constitutional varus. Clin Orthop Relat Res. 2012;470(1):45-53.

9. Bonnin MP, Saffarini M, Mercier PE, Laurent JR, Carrillon Y. Is the anterior tibial tuberosity a reliable rotational landmark for the tibial component in total knee arthroplasty? J Arthroplasty. 2011;26(2):260. e1-7.e2

10. Bonnin MP, Saffarini M, Shepherd D, Bossard N, Dantony E. Oversizing the tibial component in TKAs: incidence, consequences and risk factors. Knee Surg Sports Traumatol Arthrosc. 2016;24(8):2532-40.

11. Bonnin MP, Schmidt A, Basiglini L, Bossard N, Dantony E. Mediolateral oversizing influences pain, function, and flexion after TKA. Knee Surg Sports Traumatol Arthrosc. 2013;21(10):2314-24.

12. Mahoney OM, Kinsey T. Overhang of the femoral component in total knee arthroplasty: risk factors 
and clinical consequences. J Bone Joint Surg Am. 2010;92(5):1115-21.

13. Insall J. Total knee replacement. In: Surgery of the knee. New York: Churchill Livingstone; 1984. p. 587-695.

14. Berger RA, Crossett LS, Jacobs JJ, Rubash HE. Malrotation causing patellofemoral complications after total knee arthroplasty. Clin Orthop Relat Res. 1998;356:144-53.

15. Howell SM, Howell SJ, Kuznik KT, Cohen J, Hull ML. Does a kinematically aligned total knee arthroplasty restore function without failure regardless of alignment category? Clin Orthop Relat Res. 2013;471(3):1000-7.

16. Parratte S, Pagnano MW, Trousdale RT, Berry DJ. Effect of postoperative mechanical axis alignment on the fifteen-year survival of modern, cemented total knee replacements. J Bone Joint Surg Am. 2010;92(12):2143-9.

17. Bonnin M, Laurent JR, Parratte S, Zadegan F, Badet R, Bissery A. Can patients really do sport after TKA? Knee Surg Sports Traumatol Arthrosc. 2010;18(7):853-62.
18. Bonnin MP, Basiglini L, Archbold HA. What are the factors of residual pain after uncomplicated TKA? Knee Surg Sports Traumatol Arthrosc. 2011;19(9):1411-7.

19. Bourne RB, Chesworth BM, Davis AM, Mahomed NN, Charron KD. Patient satisfaction after total knee arthroplasty: who is satisfied and who is not? Clin Orthop Relat Res. 2010;468(1):57-63.

20. Noble PC, Conditt MA, Cook KF, Mathis KB. The John Insall award: patient expectations affect satisfaction with total knee arthroplasty. Clin Orthop Relat Res. 2006;452:35-43.

21. Niki Y, Nagura T, Nagai K, Kobayashi S, Harato K. Kinematically aligned total knee arthroplasty reduces knee adduction moment more than mechanically aligned total knee arthroplasty. Knee Surg Sports Traumatol Arthrosc. 2018;26(6): 1629-35.

22. Almaawi AM, Hutt JRB, Masse V, Lavigne M, Vendittoli P-A. The impact of mechanical and restricted kinematic alignment on knee anatomy in total knee arthroplasty. J Arthroplast. 2017;32:2133-40.

Open Access This chapter is licensed under the terms of the Creative Commons Attribution 4.0 International License (http://creativecommons.org/licenses/by/4.0/), which permits use, sharing, adaptation, distribution and reproduction in any medium or format, as long as you give appropriate credit to the original author(s) and the source, provide a link to the Creative Commons license and indicate if changes were made.

The images or other third party material in this chapter are included in the chapter's Creative Commons license, unless indicated otherwise in a credit line to the material. If material is not included in the chapter's Creative Commons license and your intended use is not permitted by statutory regulation or exceeds the permitted use, you will need to obtain permission directly from the copyright holder.

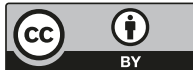

